\title{
The Performance Evaluation of 802.15.3a UWB Channel Model with Different Rake Receiver Fingers of Maximal Ratio Combiner Scheme
}

\author{
Sachin Taran \\ Electronics and Communication \\ Medicaps Group of Institution \\ Indore, India
}

\author{
Dhiraj Nitnaware \\ Electronics and Communication \\ IET DAVV \\ Indore, India
}

\begin{abstract}
In this paper we proposed an analytical method which is used for 802.15.3a UWB channel to find out the effect of rake receiver finger with Maximal ratio combining (MRC) scheme. We used a mathematical model which is derived while considering the receiver is MRC rake receiver. In this receiver we assigned weights to rake receiver finger in such a way to maximize the signal to noise ratio (SNR). With the help of maximal ratio combining (MRC) mathematical model we try to evaluate the performance result with different rake receiver finger.
\end{abstract}

\section{Keywords}

Lognormal fading, Maximal ratio combining (MRC), 802.15.3a channel model, Wilkinson's method, BPSK.

\section{INTRODUCTION}

A widely accepted UWB (Ultra Wide Band) indoor communication channel model 802.15.3a developed by channel modeling subcommittee and it is define in $[1,2]$.

According to [2] the 802.15.3a channel model UWB system exhibits high frequency selective fading and can be modeled as a discrete linear filter whose impulse response is expressed as,

$$
\mathrm{h}(\mathrm{t})=\sum_{\mathrm{l}=0}^{\mathrm{L}_{\max }-1} \beta_{\mathrm{l}} \alpha_{\mathrm{l}} \delta\left(\mathrm{t}-\tau_{\mathrm{l}}\right)
$$

The $\mathrm{L}_{\max }$ is the maximum number of resolvable multipath components (MPCs) and it is decided by the number of fingers of rake receiver or sampling resolution of receiver.

The $\alpha_{l}$ is the channel fading coefficient for the $l^{\text {th }}$ path and its

$$
\text { Transmitted Symbol }
$$

$$
\text { Received Symbol }
$$

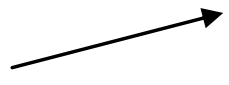

Finger\#1

Finger\#2

Finger\#3

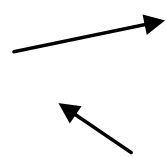

modeled as lognormal random variable, whose parameter depends on the arrival time $\tau_{l}$. The $\tau_{l}$ is arrival time of $l^{\text {th }}$ path

relative to $1^{\text {st }}$ path $\left(l=0 \& \tau_{0}=0\right.$ assumed $)$ and $\delta(t)$ is Dirac delta function.

The channel gain $\alpha_{l}$ is modeled as $\alpha_{1}=\mathrm{p}_{1} \beta_{l}$, where $p_{l}=+1$ $/-1$ with equal probability it models the random pulse inversion that can occur due to reflections it is define in [2]. The second term $\beta_{l}$ is a magnitude of $l^{\text {th }}$ MPC and it is log normally distributed for indoor channels. The parameters of lognormal random variable are mean and standard deviation. The standard deviation of fading amplitude is in the range of $3-5 \mathrm{~dB}$ given in [2] and mean value depends on the parameters of channel profile CM1, CM2, CM3, CM4 such as cluster arrival rate , ray arrival rate ,cluster decay factor, ray decay factor etc given by $[1,2]$.

These channel profile parameters such as distribution of path arrival time sequence $\tau_{l}$ and power delay profile (PDP) of the channel are chosen to follow the modified Saleh-Valenzuela (S-V) model suggested in [3].

\subsection{Maximal Ratio Combining (MRC)}

It is well known that a RAKE receiver with maximal ratio combining (MRC) is optimum when the desired signal is distorted by the additive white Gaussian noise (AWGN). Maximal-Ratio Combining, first discussed by Brennan, is the optimal form of diversity combining because it yields the maximal SNR achievable. It requires the exact knowledge of SNRs as well as the phases of the diversity signals. [4]

$\begin{array}{cc}\text { Modified with } & \text { Combined } \\ \text { Channel Estimate } & \text { Symbol }\end{array}$
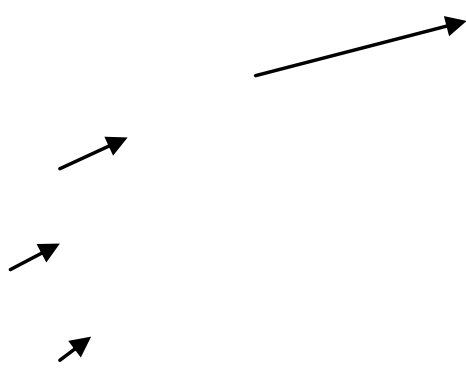

Figure 1. Maximal Ratio Combining in RAKE 
After de-spreading the received symbol from transmitter via radio channel the symbols from allocated fingers are maximal-ratio-combined to construct the "combined" symbol. The output symbols from different fingers are multiplied with complex conjugate of the channel estimate and the result of multiplication is summed together into the "combined" symbol. This is illustrated in Figure 1 and Figure2, given in [5]. One more advantage of MRC scheme that it is performance similar to minimum mean square error (MMSE) combining scheme but complexity lower than MMSE this is also proof in [6].

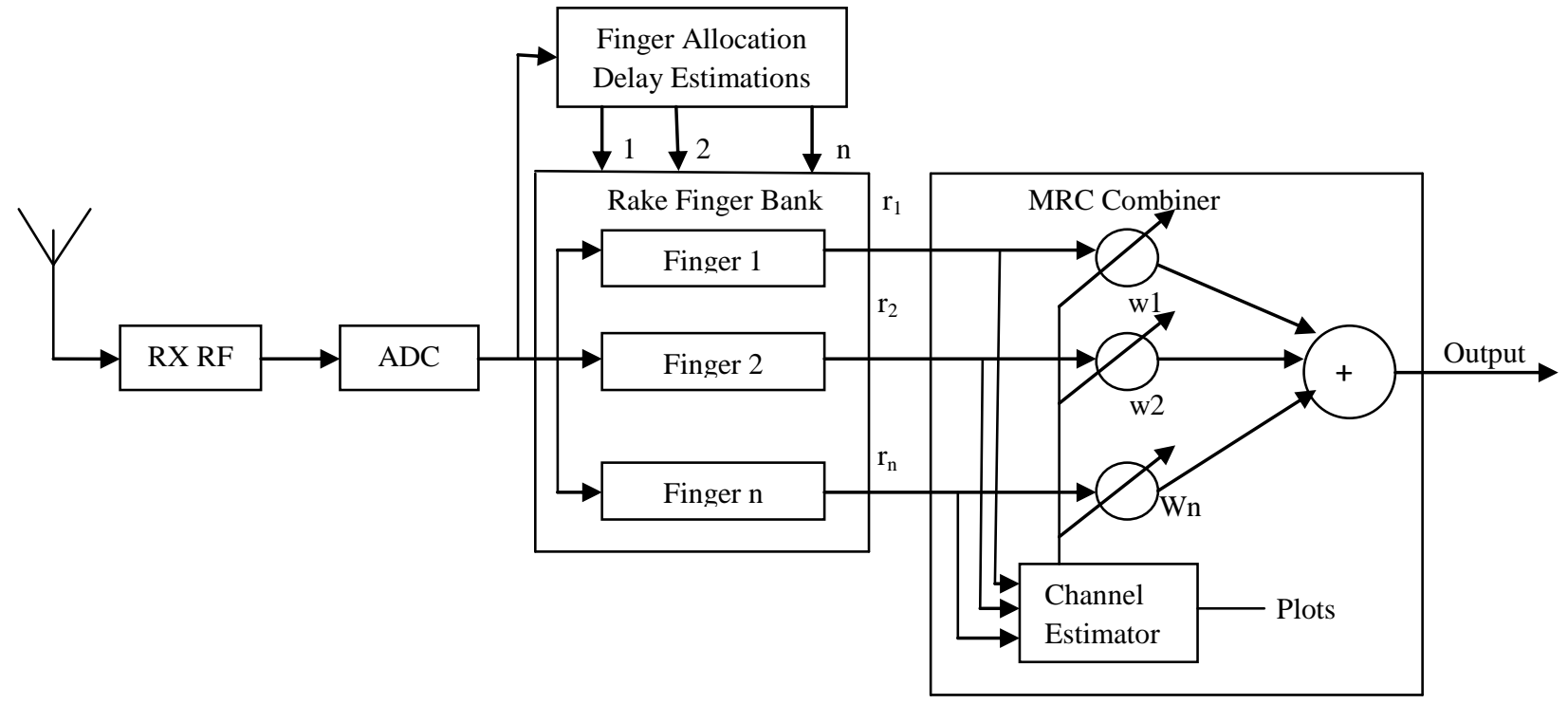

Figure 2. RAKE receiver using MRC

\section{PROBLEM FORMATION}

As in [7] they present the instantaneous signal to noise ratio (SNR) model by considering one of the channel impairments i.e. intra pulse interference (IPI) according to [7] for a realistic UWB system having a finite temporal resolution $T_{p}$ i.e. often limited by the receiver sampling frequency or the width of UWB pulse. The instantaneous signal to noise ratio (SNR) per bit can be written as

$$
\begin{array}{r}
\gamma_{i}=\frac{\mathrm{E}_{s}}{\mathrm{~N}_{0}} \sum_{l=0}^{\left[\frac{\mathrm{T}_{\mathrm{i}}}{\mathrm{T}_{\mathrm{p}}}\right]-1} \gamma_{l}^{2} \\
=\frac{\mathrm{E}_{s}}{\mathrm{~N}_{0}} \sum_{l=0}^{\left[\frac{\mathrm{T}_{\mathrm{i}}}{\mathrm{T}_{\mathrm{p}}}\right]-1}\left[\sum_{\mathrm{i} \in \mathrm{I}_{l}}\left(\beta_{\mathrm{i}} \alpha_{\mathrm{i}}\right)\right]^{2}
\end{array}
$$

From (2), the receiver seems to be a maximum ratio combining (MRC) Rake receiver with $\left[\frac{T_{i}}{T_{p}}\right]$ uniformly-spaced fingers, where the effective weighting coefficient of the $l^{\text {th }}$ finger is $\left.\sum_{\mathrm{i} \in \mathrm{I}_{l}}\left(\beta_{\mathrm{i}} \alpha_{\mathrm{i}}\right)\right]$.

In this model second summation is taken for accounting the intra pulse interference i.e. occurs between MPCs. where $\mathrm{I}_{l}$ is set given by: $\mathrm{I}_{l}=\left\{\mathrm{i} \mid l T_{p} \leq \tau_{i}<(l+1) T_{p}\right\}$ denotes the collection of multipath components (MPCs) arriving in the interval $\left[l T_{p},(l+1) T_{p}\right]$, i.e. the number of MPCs in a single finite temporal resolution but right now in this paper the impairments that we want to model is shadowing not the intra pulse interference so width of pulse is adjusted such that $T_{p}<<1$. In this case $\gamma_{l} \rightarrow \beta_{l} \alpha_{l}$ and equation (2) from the [7] can be written as

$$
\gamma_{i}=\frac{\mathrm{E}_{s}}{\mathrm{~N}_{0}} \sum_{\mathrm{l}=0}^{\mathrm{L}_{\max }-1} \alpha_{l}^{2}
$$

\section{PERFORMANCE ANALYSIS}

The major parameters that researchers generally use to figure out the performance of UWB channel are outage probability and average bit error rate (ABER). In this paper we are using the ABER

The process of finding the ABER given in [8], the average bit error rate can be calculated by averaging the conditional BER which is $P\left(\gamma_{i}\right)$ over the probability density function (PDF) of instantaneous SNR i.e. $f\left(\gamma_{i}\right)$ as

$$
P_{e}=\int_{0}^{\infty} P\left(\gamma_{i}\right) f\left(\gamma_{i}\right) d \gamma_{i}
$$

The conditional BER for different modulation scheme are given in [9]. According to [9] best modulation scheme for UWB belongs to antipodal category. The antipodal signal scheme in which multiplication of two transmitted symbol is -1 and it is Binary phase shift keying (BPSK).

The classic binary phase shift key (BPSK) can be presented using two antipodal Gaussian pulses as shown in Figure 3 given in [9]. The transmitted binary baseband phase shift modulated information signal $\mathrm{X}(\mathrm{t})$ can be presented as

$$
\mathrm{X}(\mathrm{t})=d_{j} * \mathrm{P}(\mathrm{t})
$$

Where $P(t)$ represents the UBW pulse waveform, $j$ represents the bit transmitted (' 0 ' or ' 1 ') and

$$
d_{j}= \begin{cases}-1, & j=0 \\ 1, & j=1\end{cases}
$$




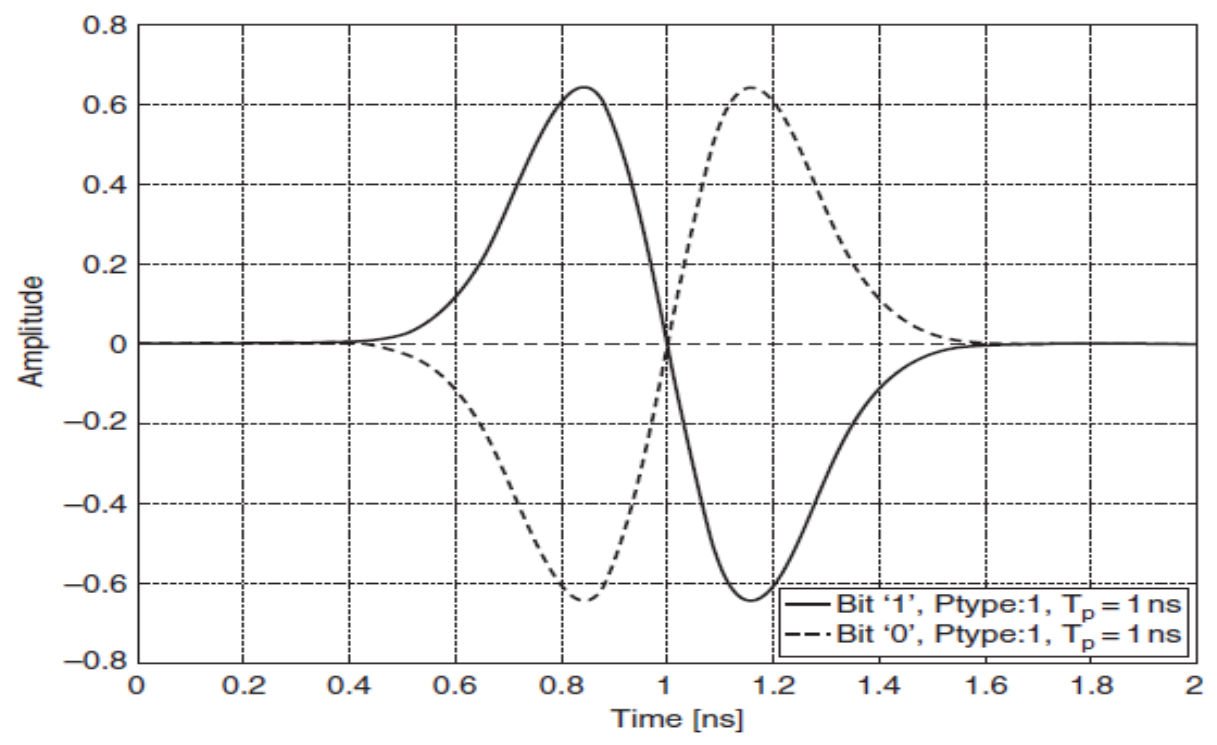

Figure 3. BPSK pulse shapes for ' 1 ' and '0' bits

The conditional BER for fixed set of $\beta_{l}$ for BPSK is given as

$$
P\left(\gamma_{i}\right)=Q \sqrt{2 \gamma_{i}}=\mathrm{Q}\left(\sqrt{\frac{2 \mathrm{E}_{\mathrm{b}}}{N_{0}}}\right)
$$

Where,

$$
\gamma_{i}=\frac{E_{\mathrm{b}}}{N_{0}}=\text { signal to noise ratio. }
$$

For calculating the ABER we require the PDF of instantaneous SNR $f\left(\gamma_{i}\right)$. There are many methods for calculating the PDF of instantaneous SNR. A major section of research in the field of UWB has been done for finding the accurate PDF of their instantaneous SNR model. In [7] they have suggested three methods for approximating the PDF of instantaneous SNR model according to severances of fading. The distributions used in [7] to approximate the PDF of $\gamma_{i}$ are coxian, Lognormal, Gamma and parameters of these approximation are find out using least square error criteria by minimizing the mean square error. Our process for finding the PDF of instantaneous SNR is little bit different and it is best according to our model. In this paper we have computed the PDF of term lognormal random variables and estimation of distributions can be given by [10]. They have suggested three methods for the estimation of distribution. The method which we are using in this paper is Wilkinson's method. From Wilkinson's method the PDF of instantaneous SNR given as

$$
f\left(\gamma_{i}\right)=\frac{1}{\gamma_{1} \sqrt{2 \pi \sigma_{z}^{2}}} e^{\left[-\frac{\left(\ln \left(\gamma_{1}\right)-\mu_{z}\right)^{2}}{2 \sigma_{z}^{2}}\right]}
$$

Parameter of this PDF is mean $\left(\mu_{\mathrm{z}}\right)$ and standard deviation $\left(\sigma_{\mathrm{z}}\right)$ which comes from [10].

\section{NUMERICAL RESULT: FRAMEWORK VALIDATION}

These results obtain for different number of rake receiver finger. Number of rake receiver finger is $\mathrm{L}=10,8,6$ respectively. To obtain these results the range of $\operatorname{SNR}\left(\frac{\mathrm{E}_{s}}{\mathrm{~N}_{0}}\right)$ is 1 to $10 \mathrm{~dB}$.

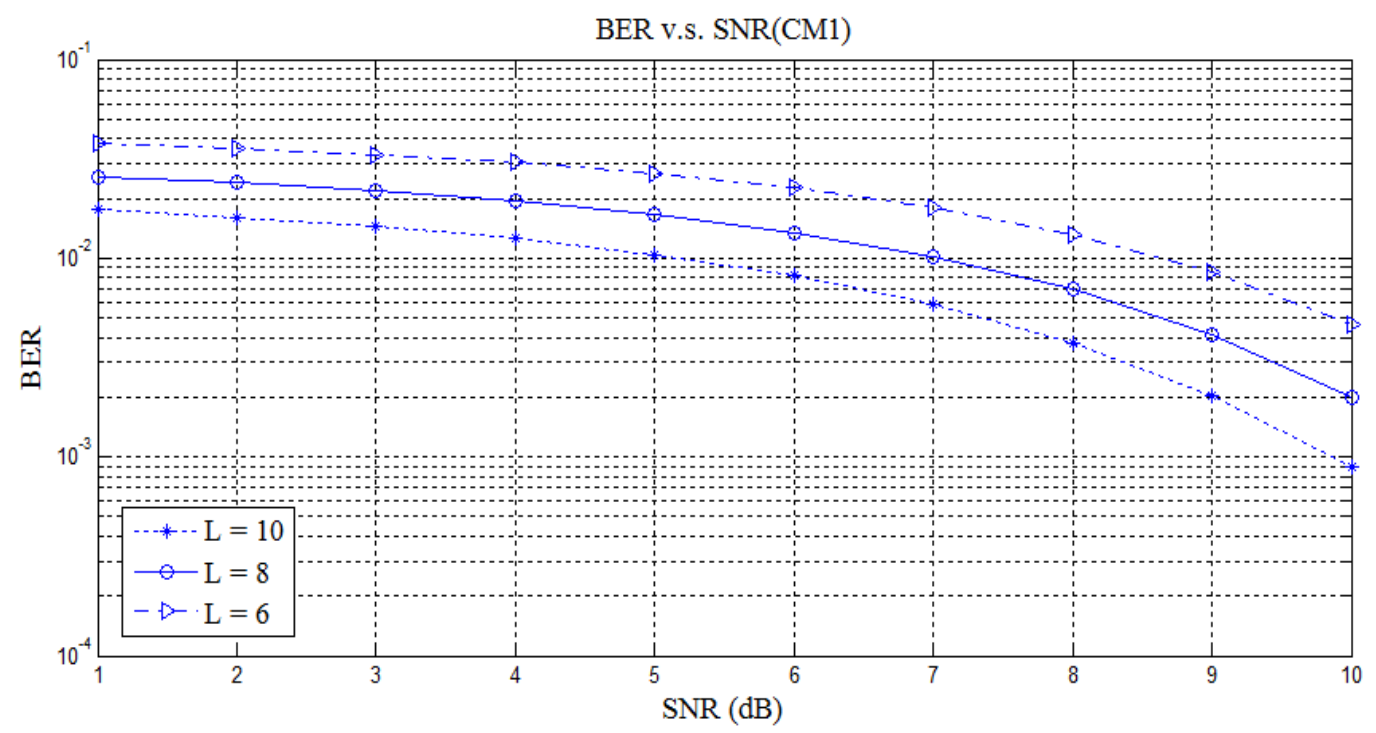

Figure 4. BER Performance of the IEEE 802.15.3a UWB channel CM1 with RAKE receiver fingers $L=6,8$, and 10 . 
The above result is obtained for channel model 1 . This channel model is of a line of sight (LOS) case with the transmitter and the receiver antenna being separated by a distance in the range $(0-4 \mathrm{~m})$. The parameters used for these result are given in [3].
From the figure it is clear that the average bit error rate (ABER) for 10 finger rake receiver case of CM1 is below $10^{-3}$ at $\mathrm{SNR}$ value of $10 \mathrm{~dB}$.

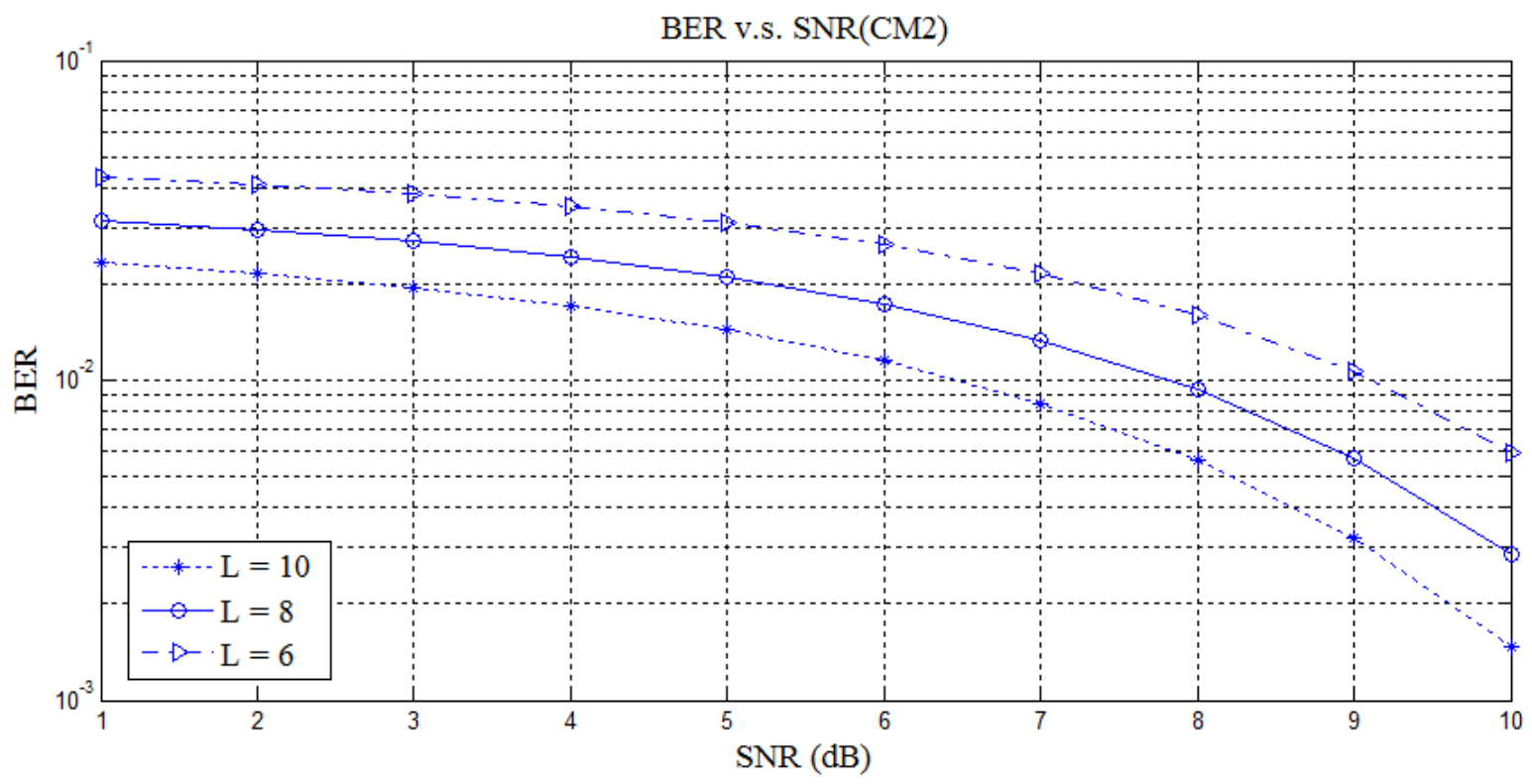

Figure 5. BER Performance of the IEEE 802.15.3a UWB channel CM2 with RAKE receiver fingers $L=6,8$, and 10 .

The above result is obtained for channel model 2. This channel is a model for a non line of sight (NLOS) case with antenna separation being in the range $(0-4 \mathrm{~m})$. The parameters used for these result are given in [3]. From the figure it is clear that the average bit error rate (ABER) for 10 finger rake receiver case of CM2 is near of $10^{-3}$ at SNR value of $10 \mathrm{~dB}$.

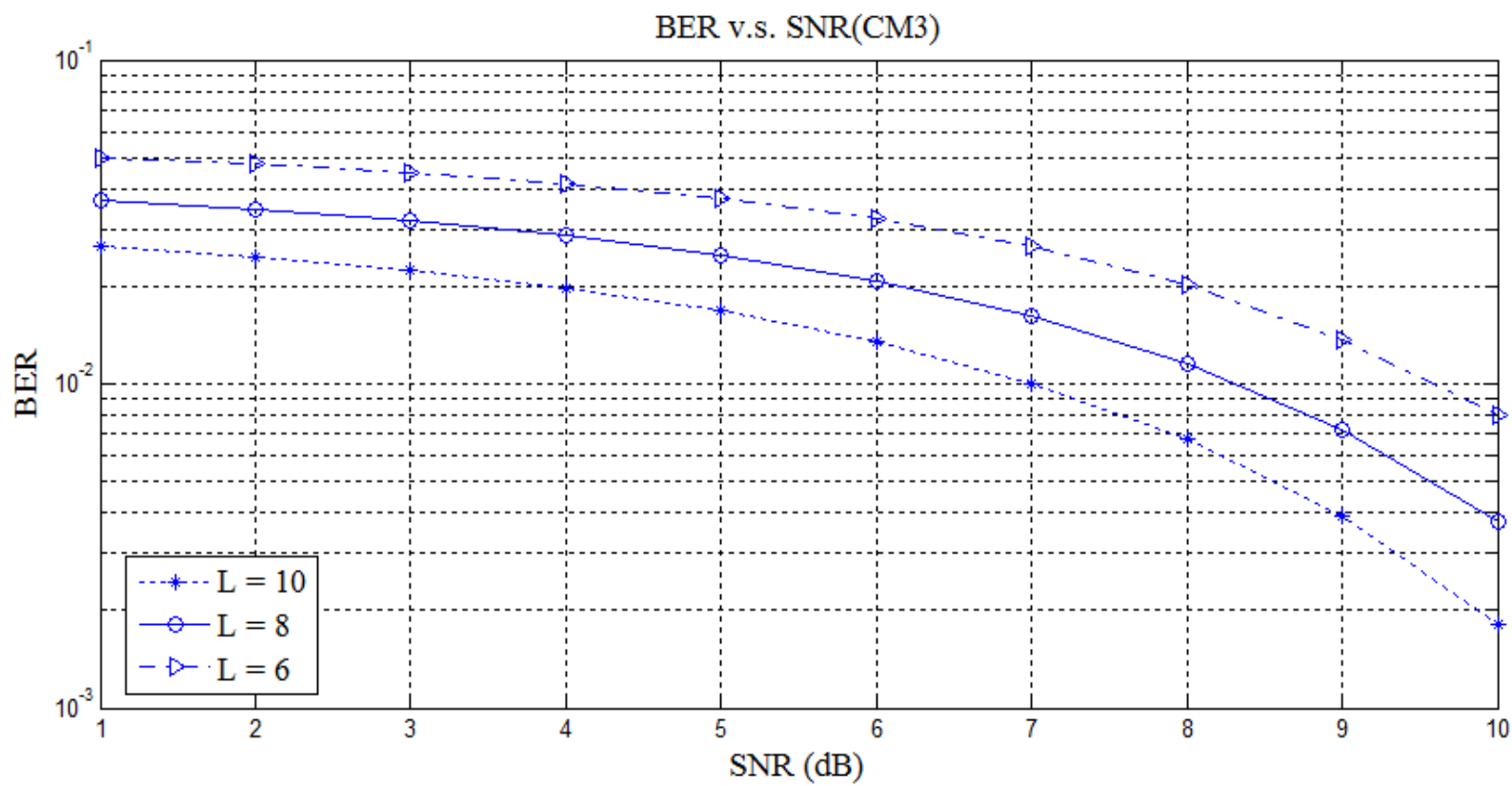

Figure 6. BER Performance of the IEEE 802.15.3a UWB channel CM3 with RAKE receiver fingers $L=6,8$, and 10 .

The above result is obtained for channel model3. This channel is a model for a non line of sight (NLOS) case with antenna separation being in the range $(4-10 \mathrm{~m})$. The parameters used for these result are given in [3]. From the figure it is clear that the average bit error rate (ABER) for 10 finger rake receiver case of CM3 is above of $10^{-3}$ at SNR value of $10 \mathrm{~dB}$. 


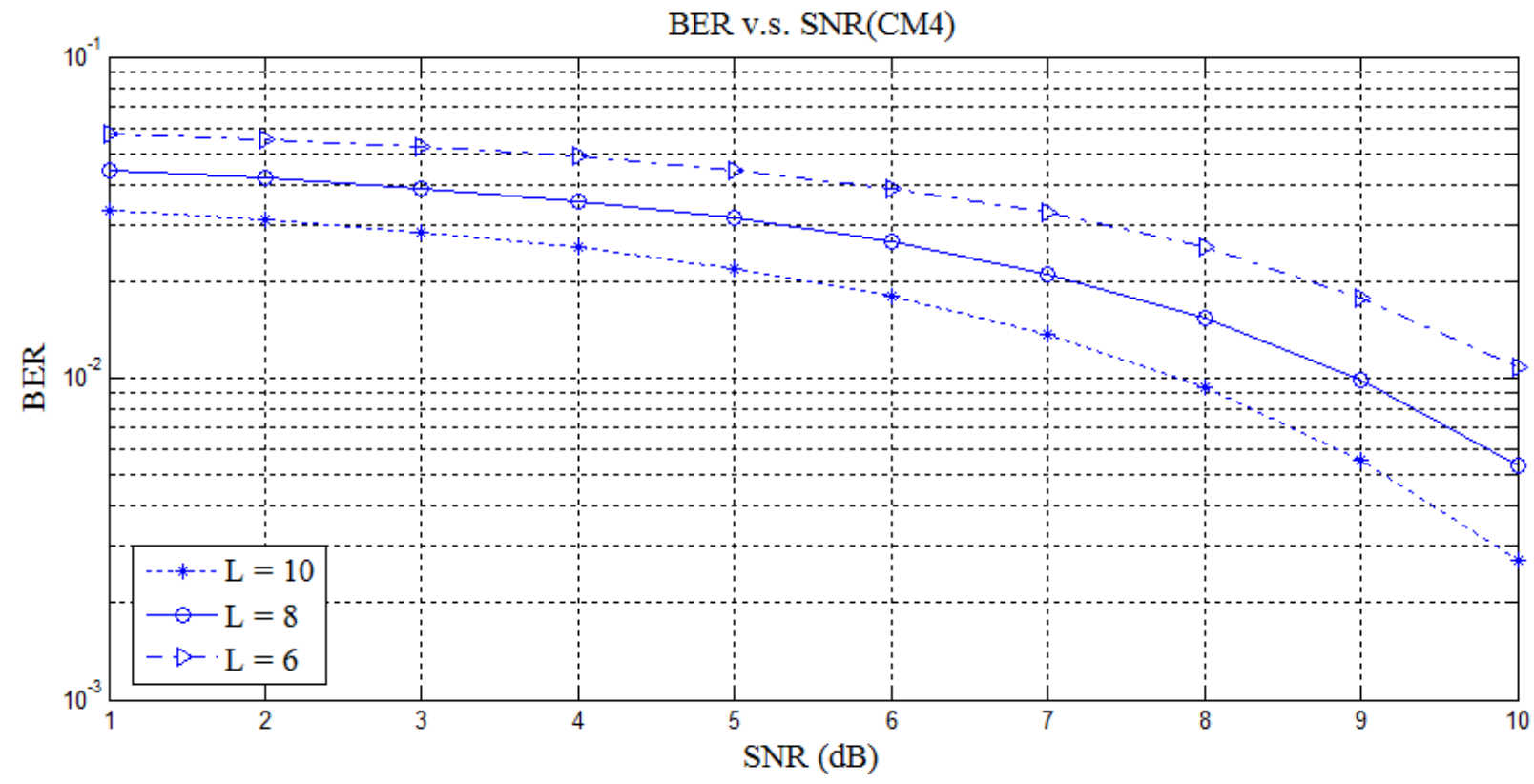

Figure 7. BER Performance of the IEEE 802.15.3a UWB channel CM4 with RAKE receiver fingers $L=6,8$, and 10

The above result is obtained for channel model 4. This channel is a model for a extreme NLOS multipath channel to fit a $25 \mathrm{~ns}$ RMS delay spread. The parameters used for these result are given in [3]. From the figure it is clear that the average bit error rate (ABER) for 10 finger rake receiver case of CM4 is in the mid of 10-2 and 10-3 at SNR value of $\mathrm{dB}$. But from all of the above results minimum BER is obtained for 10 finger rake receiver and maximum BER is obtained for 6 finger rake receiver.

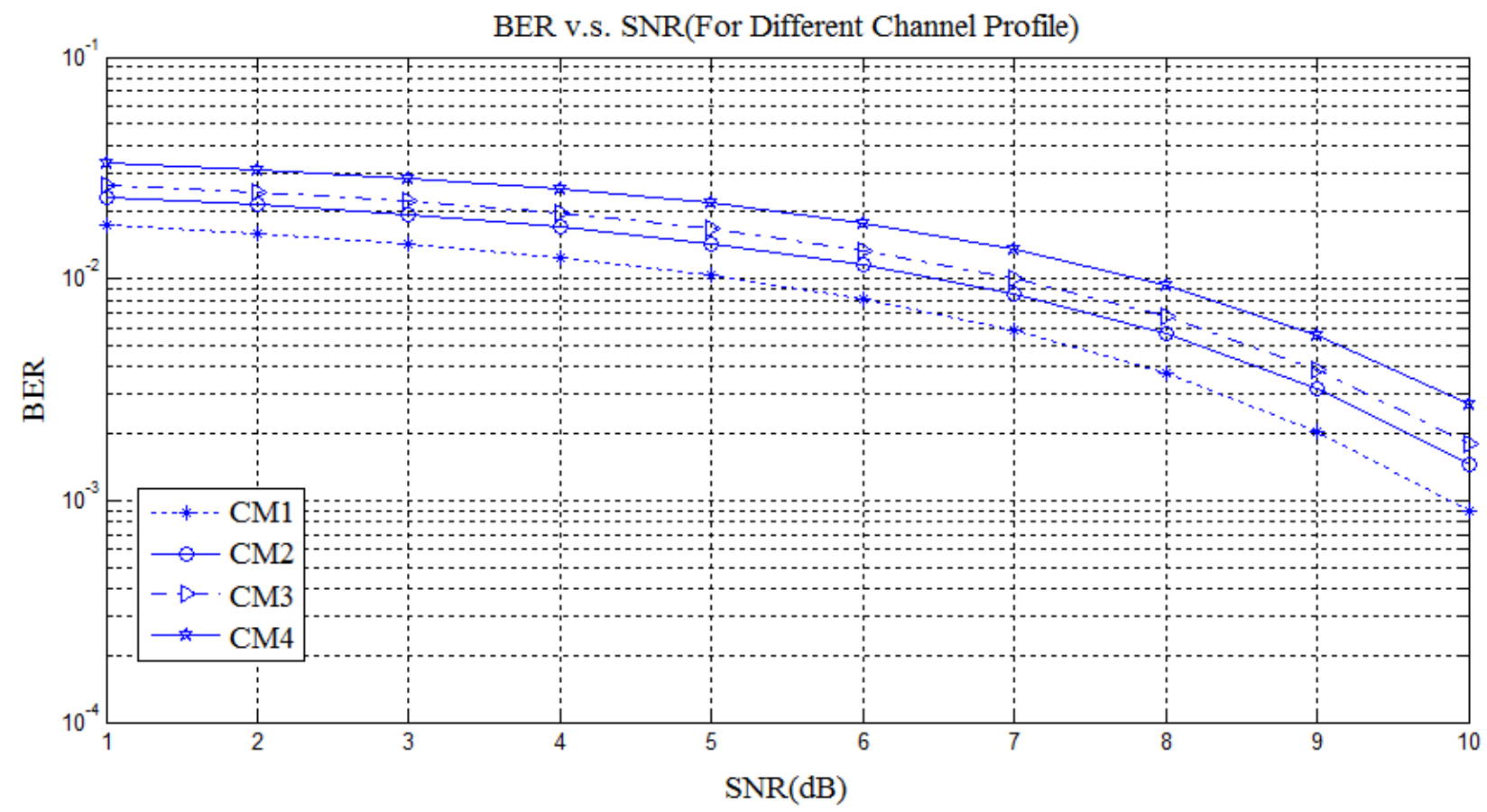

Figure 8. BER Performance of the IEEE 802.15.3a UWB channel CM1, CM2, CM3, CM4 with RAKE receiver fingers L $=10$.

In these result we compare four channel profiles. To obtain these results the number of fingers taken for rake receiver is ten. From the figure it is clear that the average bit error rate (ABER) is minimum for CM1 is near about $10^{-3}$ and maximum for CM4 it is near about $10^{-2}$ at SNR value of $10 \mathrm{~dB}$. 


\section{CONCLUSION}

We proposed an analytical method which is too easy to verify the performance of 802.15.3a channel model with MRC receiver and different rake receiver finger. In this analytical method for calculation of ABER we require PDF of instantaneous SNR which is estimated from the Wilkinson's method. Finally we got computable formula for ABER. We derived our results for different channel profile with different rake receiver finger. The results clearly shows that if number of finger is increased then number of MPCs will also increased and this will turn increase the SNR at the receiver. We know that ABER and SNR inversely related to each other, it can also inferences from the $Q$ function which is a function of SNR. So increasing of SNR turn to reduce the ABER. One interesting research topic that can be extended from this work is to apply this method to any multipath fading models with other general random arrival process for clusters and rays, such as the IEEE 802.15.4a outdoor channel model.

\section{REFERENCES}

[1] A. F. Molisch, J. R. Foerster, and M. Pendergrass, "Channel models for ultrawideband personal area networks," IEEE Wireless Commun., vol. 10, pp. 14-21, Dec. 2003.

[2] J. Foerster, Ed., "Channel Modeling Sub-committee Report Final," IEEE, Document IEEE P802.15-02/490r1SG3a, 2003.
[3] A. A. M. Saleh and R. Valenzuela, "A statistical model for indoor multipath propagation," IEEE J. Select. Areas. Commun., vol. SAC-5, no. 2, pp. 128-137, Feb. 1987.

[4] Samuel C. Yang, CDMA RF System Engineering, Norwood MA, USA, Artech House Inc., 1998, 280 pp.

[5] Mikko Järvelä, RAKE training slides by Nokia, 03.04.2001, Oulu.

[6] Shiwei Zhao and Huaping Liu, Member, IEEE on the Optimum Linear Receiver for Impulse Radio Systems in the Presence of Pulse Overlapping IEEE communications letters, vol. 9, no. 4, April 2005.

[7] A.-R. Chadi, "Performance analysis of UWB systems over the IEEE802.15.3a channel model," IEEE Trans. Commun., vol. 59, no. 9, pp. 2377-2382, Sep. 2011.

[8] John G. Proakis, Masoud Salehi, Digital Communications, 5th ed. New York: McGraw-Hill, 2008, ch. 14.

[9] Huseyin Arslan, Zhi Ning Chen, Maria-Gabriella Di Benedetto, Ultra wideband wireless communication, 3rd ed. New John Wiley \& Sons Ltd, 2006, ch. 4.

[10] N. C. Beaulieu, A. A. Abu-Dayya, and P. J. Mclane, "Estimating the distribution of a sum of independent lognormal random variable," IEEE Trans. Commun., vol. 43, pp. 2869-2873, Dec. 1995. 